\title{
Pediatric Practice on Parents' Presentation: ARM Luthful Kabir (ed)
}

\author{
Published by Dr. Nazneen Kabir, Dhaka, Bangladesh, ISBN:978-984-33-2423-8, Edition: \\ First, Price: TK. 1,000, 20 US\$
}

\section{Suresh Gupta}

Received: 22 May 2012 / Accepted: 22 May 2012 /Published online: 7 June 2012

(C) Dr. K C Chaudhuri Foundation 2012

The blue cover of the book with a sketch of parents with a child reflect the theme and concept of this work. This book spread over 900 pages is distinct and different and stands out in the crowd with its unique way to deal with this subject. The book addresses the constellations of symptoms with which children are brought by their parents to the pediatricians. The approach seems to be more true-life, practical and pragmatic at the same time. The book has 74 scenarios which are explained with 182 real case examples. The addition of 536 pictures makes this book very interesting and engaging. The tabular format along with cluster clues as highlighter, makes it decipherable and user friendly. Through the 74 scenario, the author discusses the common differential diagnosis along the line of history, physical examination and investigations. Though the content of the book reflects the childhood disease pattern and cultural background of Bangladesh, however a similar pattern is seen in South East Asia including India. So the manuscript seems to be useful for Indian pediatricians too. A significant emphasis has been placed on growth monitoring and parental counseling. The author has also included art of history taking and physical examination along with growth charts, immunization; schedule and nutritional advice. The book has limited itself to initial treatment and the detailed management protocols need to be taken care of. Though the book is humbly priced at $20 \$$ but its weight $(2 \mathrm{~kg})$ may make it difficult to carry everywhere. Overall it is a useful resource for pediatricians while working in indoor wards, emergency or office practice.
S. Gupta $(\bowtie)$

Department of Pediatric Emergency Medicine and Acute Care, Institute of Child Health, Sir Ganga Ram Hospital, New Delhi 110060, India

e-mail: sureshgupta62@gmail.com 